\title{
Propagation and Phase Reconstruction of ocular wavefronts with SAR techniques
}

\author{
Carlos Illueca \\ Dept. Optica, Universidad de Alicante \\ P.O. Box 99 \\ 03080 Alicante (Spain)
}

Jessica Rouarch, Julian Espinosa, Juan José Miret, David Mas, Jorge Pérez and

\begin{abstract}
In this communication, we report a new method to determine the wavefront aberration function of personalized eyes. We obtain corneal surface data and axial lengths for different eyes. With these data, we construct a diffractive model of a human eye, which permits calculation of propagated light distributions at any distance inside the eye. We obtain the field at the exit of crystalline lens and extract the phase information. To unwrap this phase we use a path-following method and with the reconstructed phase we obtain the wavefront aberration function that we analyze using Zernike polynomials. The method allows linking between field patterns and wavefront surfaces that are softer and easier to analyze. To demonstrate the possibilities of the technique we have chosen five eyes which present different level of corneal irregularities, from quasi-spherical cornea to a very deformated one.
\end{abstract}

Keywords: Optical aberrations, Zernike coefficients, human eye model, Fresnel integral calculation, Phase unwrapping. 
"Special Issue in Physiological Optics"

\section{Introduction}

During the last years, we have seen an important increase of refractive surgery, which has created a clinical need to better understand the optics of the eye. Scientists have recently developed new techniques to measure the aberrations in the human eye. Most of them consist of analysing the wavefront aberration function $W(x, y)$ that is obtained through laser-ray-tracing techniques or Hartmann-Shark interferometers [1-4]. Optical performance of the eye is governed by the combination of two principal refractive elements, the anterior surface of the cornea and the crystalline lens. How the aberrations of the cornea and the lens contribute to the final quality of the image on the retina, is a central problem in physiological optics [5].

Additionally to experimental measures, realistic mathematical models are of great importance for studying the optical performance of the eye. Much surgery on the eye is not reversible. Thus, a priori knowledge of the problem is necessary.

Therefore, the aim of this study is to develop an algorithm, based on personalized model of ocular system and by means of the transversal propagation of the light through the visual system. This model may permit evaluation of the quality of the retinal image and especially to determination of the wavefront aberration function, $W(x, y)$. Calculation of the $W(x, y)$ function requires obtaining the wavefront of the field, what implies to extract the phase of a complex field, and thus work whit inverse trigonometric functions. This produces an ambiguous phase whit values between $-\pi$ and $\pi$. The process of reconstruction the true phase is called "phase unwrapping" and is widely used in synthetic aperture radar (SAR) interferomery [6]. We have adapted and implemented some of the SAR techniques to our problem. Finally, estimation and 
"Special Issue in Physiological Optics"

quantification of optical aberrations has been done through Zernike polynomial expansion of $W(x, y)[7]$.

The first task of the method is to estimate the personalized data of each eye, hence we make a corneal topography and a biometry. The crystalline lens cannot be accessed from outside and it can only be specified through indirect measures. For this reason we implement a realistic model of a crystalline lens based on the geometry of Dubbelman crystalline [8], with age dependence.

Light pattern in any plane inside the second chamber of the eye is calculated through angular spectrum propagation $[9,10]$. We obtain the $W(x, y)$ function by extracting the wavefront of the field at the exit plane of the crystalline lens. Before that, we need to unwrap the wavefront using a path-following method [11]. We applied the algorithm for different eyes, and analyze its respective Zernike coefficients.

\section{Method}

The objective is to calculate the light pattern at the exit crystalline's plane, and foron this pattern, construct the wavefront aberration function. Assuming that the incoming beam is known, we determinate the actuation of the cornea on the beam, next, we propagate the result to the crystalline lens, and then we consider the actuation of the crystalline lens.

To know the influence of the cornea and the crystalline lens, we consider them as phase elements and therefore we need to calculate the phase delay introduced by its on the total field. Transmittance of those elements is calculated through their morphologies. Anterior chamber length $\left(z_{A C}\right)$ and axial length $\left(z_{A L}\right)$ of each eye are determined with a non-contact biometer. 
"Special Issue in Physiological Optics"

Propagation implies working with convergent light patterns, so we have used an algorithm previously developed by us [10].

We show a scheme of this process in Fig.1, where you can see also the Gaussian reference sphere that we have chosen.

'[Insert figure 1 about here]'

\subsection{Transmittance of the cornea and the crystalline lens}

With regard to corneal morphology, we assume that the cornea is a convex-concave lens. The anterior surface is reconstructed from topographic data taken with a Zeiss Humphrey ATLAS ${ }^{\odot}$ topographer [12]. The posterior corneal surface is described by the equation

$$
x^{2}+y^{2}+\left(1+x^{2}\right) z^{2}-2 R_{2} z=0
$$

where $R_{2}$ and $x_{2}$ are the axial radius of curvature and the asphericity parameters, respectively and both depend on the age and $x, y$ and $z$ are special coordinates, the $z$-axis being the optical axis. The parameters which describe the posterior corneal surface are those estimated by Dubbelman et al. [13]

$$
\begin{aligned}
& R_{2}=6.6-0.005 \cdot \text { age } \\
& x_{2}=-0.1-0.007 \cdot \text { age }
\end{aligned}
$$

where age is the age of the subject in years.

With this corneal morphology data, we are ready to know the transmittance of the cornea [14]. 
"Special Issue in Physiological Optics"

The crystalline lens is a complex graded index structure that changes with age state. There are many models of crystalline lens. We developed a model based on Dubbelman crystalline lens [8]

$$
\begin{aligned}
& R_{1}=12.9-0.03 \cdot \text { age } \\
& x_{1}=-6.4+0.03 \cdot \text { age } \\
& R_{2}=-6.2+0.012 \cdot \text { age } \\
& x_{2}=-6+0.07 \cdot \text { age } \\
& \text { thick }=3.46+0.013 \cdot \text { age }
\end{aligned}
$$

where $R_{1}, R_{2}$ and $x_{1}, x_{2}$ are the axial radii of curvature and the asphericity parameters of the first and second surface of the crystalline, respectively. Furthermore, we have completed the model with incorporation of an index gradient, the characteristic of this crystalline are described in [15].

\subsection{Propagation through ocular media}

We need to propagate the light beam that exits the cornea through the anterior chamber. The distance of propagation is very short (about $4 \mathrm{~mm}$ ) and furthermore it is of the same order that pupil aperture. In these conditions calculation of the light pattern through the Fresnel integral does not guarantee enough accuracy. For this reason we have decided to use Rayleigh-Sommerfeld approximation. It may be summarize as follow.

Let us consider an isotropic, homogeneous and non-dispersive medium covering the half-space $z \geq 0$. Let us consider an entrance pupil of circular shape at the plane $z=0$ illuminated by a normally incident plane wave of wavelength $\lambda$. The generated optical field at a distance $z$, evaluated at the spatial coordinates $\left(x_{z}, y_{z}\right)$ can be described by a complex function $u_{z}$ given by [16] 
"Special Issue in Physiological Optics"

$$
\begin{aligned}
u_{z}\left(x_{z}, y_{z}, z\right) & =\int_{-\infty}^{+\infty} \tilde{u}_{0}(\xi, \eta) \exp \left(i \frac{2 \pi}{\lambda} z\left[1-(\lambda \xi)^{2}-(\lambda \eta)^{2}\right]^{1 / 2}\right) \\
& \times \exp \left[i 2 \pi\left(\xi x_{z}+\eta y_{z}\right)\right] d \xi d \eta
\end{aligned}
$$

where $\tilde{u}_{0}(\xi, \eta)$ is the Fourier transform of the field at $z=0$ evaluated at the spatial frequencies $(\xi, \eta)$. Note that equation (4) is an exact solution of the Helmholtz wave equation, and not an approximation as the Fresnel integral. Note also that this equation is symmetric with respect to both $x_{z}$ and $y_{z}$ and their respective conjugated variables $\xi$ and $\eta$. Therefore, from now on we shall use one-dimensional notation in order to facilitate the equations.

Let us consider an input signal of size $\Delta x_{0}$ that has been discretized in $N$ samples. Discretization of equation (4) provides

$$
\left(u_{z}\right)_{\tilde{m}}=\operatorname{DFT}^{-1}\left\{\exp \left[i \frac{2 \pi}{\lambda} z\left(1-\left(\lambda^{2} \frac{\tilde{m}^{2}}{\Delta x_{0}^{2}}\right)^{1 / 2}\right)\right] \operatorname{DFT}\left[u_{0}\left(\frac{m \Delta x_{0}}{N}\right)\right]\right\} \text {. }
$$

where $m$ and $\tilde{m}$ are discrete indices in space and Fourier domains respectively, DFT is discrete Fourier transform and $\Delta x_{0}$ is the size of the object support window.

Finally we have to take into account that the beam that exits the cornea is convergent. If we assume that it converge to a positive distance $z_{c}$, the above equation can be reformulated as

$$
\left(u_{z}\right)_{\tilde{m}}=\operatorname{DFT}^{-1}\left\{\exp \left[i \frac{2 \pi}{\lambda} z\left(1-\left(\lambda^{2} \frac{\tilde{m}^{2}}{\Delta x_{0}^{2}}\right)^{1 / 2}\right)\right] \operatorname{DFT}\left[u_{0}\left(\frac{m \Delta x_{0}}{N}\right)\right] \exp \left(-i \pi \frac{m^{2} \Delta x_{0}^{2}}{\lambda N^{2}} \frac{1}{z_{c}}\right)\right\}
$$

In reference [17] we have analyzed this algorithm for propagation of convergent beams in free-space, and we have determined the conditions (relations between $N, z_{c}, z, \lambda$ and $\Delta x_{0}$ ) that guarantee that the field (phase and amplitude) is calculated without 
"Special Issue in Physiological Optics"

aliasing. In [6] we apply the algorithm for evaluating the image quality produced by an aphakic eye.

With all these ingredients we construct a personalized eye model and we are able of calculate the propagated light distribution in any plane inside the eye. This method has been validated as statistically [18] as with individual predictions [10].

Furthermore, recently, in [19] we have established a technique that reduce considerably the number of samples needed in our calculations without introducing aliasing. This implies important savings in computer memory requirements and in computing time, thus allowing implementation of the algorithm in real-time processes.

\subsection{Wavefront aberration of the eye}

From expression (6) it is theoretically possible to access to all amplitude and phase information of the optical field. Unfortunately, phase information presents very fast oscillations because of phase ambiguities in $-\pi$ to $\pi$ jumps that may not be of easy mathematical analysis. One of the applications that may need a soft phase distribution are the reconstruction of the phase aberration function.

The first step in obtaining the aberration function consists of defining a Gaussian reference sphere, centered on the intersection of the retinal plan and the optical axis being the radii of such surface the distance between the second surface of the crystalline lens and the retinal plane.

Propagation of the light distribution up to the crystalline exit plane produces a field described as follows:

$$
U(x, y)=|A(x, y)| e^{j k \phi(x, y)}
$$

where $|A(x, y)|$ is the amplitude of the field and $\phi(x, y)$ the phase. 
"Special Issue in Physiological Optics"

The aberration function is determinated as the difference optical path between the Gaussian sphere and the phase's field $\phi(x, y)$. Therefore, the only problem left is the phase extraction. Unfortunately we can only obtain the wrapped phase $\psi(x, y)$ from the phase-extraction operation,

$$
\psi(x, y)=\arctan [\mathfrak{I}(U(x, y)), \mathfrak{R}(U(x, y))]
$$

where $\mathfrak{R}$ and $\mathfrak{I}$ refer to the real and imaginary parts, and arctan is the four-quadrant arc tangent operator where $-\pi<\psi(x, y) \leq \pi$.

'[Insert figure 2 about here]'

Reconstruction and disambiguation of the phase is calling phase unwrapping. Thus, two-dimensional (2-D) phase unwrapping is the process of attempting to recover unambiguous phase values from a 2-D array of wrapped phase values known. In figure 2a we show the central region of the wrapped phase of the field that corresponds to one of the studied eyes, concretely the eye 1 , and in the $2 b$ the recovered phase distribution. Phase unwrapping is a recurrent topic in the bibliography. There are four major categories of methods: least-squares method is based on minimizing the squared error between the observed and the estimated image [20, 21], model-based method assumes that the image to be unwrapped can be described by some model, usually a polynomial surface $[22,23]$, Bayesian-based method considers the problem in the context of Bayesian restoration, and the knowledge about the surface to be unwrapped is then given by a prior distribution [24], and path-following based method[11, 25].

In this study, we implemented an algorithm of the last category. These algorithms consist of resolving the equation

$$
\phi(x, y)=\int_{C} \nabla \phi . d r+\phi\left(x_{0}, y_{0}\right)
$$


"Special Issue in Physiological Optics"

where $\mathrm{C}$ is any path in our domain $\mathrm{D}$ connecting the points $\left(x_{0}, y_{0}\right)$ and $(x, y)$, and $\nabla \phi$ is the gradient of $\phi$.

Obviously, we have to deal with sampled data, and furthermore, instead of the values of the true phase $\phi_{i, j}$ we only have the discrete values of the wrapped phase function $\psi_{i, j}$ :

$$
\psi_{i, j}=\phi_{i, j}+2 \pi k, \quad \mathrm{k} \text { an integer }
$$

If the true phase differences between adjacent samples in our image are less than $\pi$, this is equivalent to the requirement that the sample phase is not aliased; we can obtain an estimate of the true gradient by wrapping the discrete gradient of wrapped phase [26]. Then, the problem is just to found the unwrapped phase by a discrete integration of the equation (9).

But, because of noise and discontinuities in the phase, there are singular points called phase residues. A closed contour integral of the estimated phase gradient around each residue does not vanish, thus the wrapped phase gradient becomes path dependent and an ambiguity arises when you try to sum up the integral (9).

Signs and the magnitudes of this contour integral define a charge for every residue. By analogy with the residues encountered in complex variable contour integration, we balanced these "charges" by imposing integration barriers called branch cut that must not be crossed during the unwrapping process.

In this study, between the different types of path-following methods, we implemented in our propagation algorithm that proposed by Goldstein in [11]. We choose this solution because the selection of branch cuts according to the residues is one of the most optimal and furthermore very fast.

\section{Results}


"Special Issue in Physiological Optics"

For test the implementation of the unwrapping algorithm in our method we have studied five eyes which present different levels of corneal irregularities, from quasi-spherical cornea (eyes 1 and 2) to a very deformed cornea (eye 4). We also have chosen a hyperopic eye (eye 3) and the same eye after a Lasik operation (eye 3 post). In figure 3 we show the topographies of the eyes 1 and 4 . Surface irregularities of the eye 4 have been an excellent trial for the unwrapping process.

'[Insert figure 3 about here]'

For each eye we have supposed a pupil diameter of $5 \mathrm{~mm}$ and $\lambda=555 \mathrm{~nm}$, wave front aberration function was obtained and its Zernike polynomial expansion calculated. The results are summarized in the table 1 and in the figure 4.

'[Insert table 1 about here $] '$

'[Insert figure 4 about here]'

The values of the normal eyes (1, 2 and 3) show good agreement with recently published recompilation of aberrometry measurements [27]. We can observe that, although ammetropia has been corrected, the Lasik ablation has increased considerably the Zernike coefficients (for example the RMS increases form $0.04 \mu \mathrm{m}$ to $0.10 \mu \mathrm{m}$ ). Evidently the eye 4 shows very large aberrations, with a RMS $=0.37 \mu m$, one order of magnitude superior to normal eyes.

\section{Conclusion}

We have described a complete algorithm to calculate the wavefront aberration function of personalized eyes. The personalization is accomplished through topographic and biometric data.

The algorithm consists of numerical evaluation of the transmittance of cornea and crystalline lens and the application of a modified spectrum propagation method to 
"Special Issue in Physiological Optics"

obtain light pattern distributions inside the eye. It incorporates an unwrapping technique to reconstruct the phase of the calculated complex field and thus determine the aberration function.

We have studied five eyes which present different level of corneal irregularities. The results of the normal eyes are totally comparable with bibliography dates, we have also compared an eye before and after of a Lasik ablation where we observe an appreciably rising of the aberrations.

\section{Bibliography}

[1] J. Liang, B. Grimm, S. Goelz, et al., J. Opt. Soc. Am. A 111949 (1994).

[2] J. Liang and D.R. Williams, J. Opt. Soc. Am. A 142873 (1997).

[3] R. Navarro, E. Moreno-Barriuso and C. Dorronsoro, J. Opt. Soc. Am. A 152522 (1998).

[4] S. Marcos, L. D1' az-Santana, L. Llorente, et al., J. Opt. Soc. Am. A 191063 (2002).

[5] P. Artal and A. Guirao, Opt. Lett. 231713 (1998).

[6] Dennis C. Ghiglia and Mark. D. Pritt, Two-Dimensional Phase Unwarpping: Theory, Algorithms and Software (John Wiley \& Sons, New York, 1998).

[7] American National Standards for Ophthalmics -Methods for Reposting Optical Aberrations of Eyes, ANSI Z80.28. New York, NY, American National Standards Institute, Inc, 2004.

[8] M. Dubbelman and G.L. Van Der Heijde, Vision Res. 411867 (2001)

[9] D. Mas, J. Perez, C. Hernandez, C. Vázquez, J.J. Miret, C. Illueca, Opt. Com. 227 $245(2003)$.

[10] J. Perez, D. Mas, C. Illueca, J.J. Miret, C. Vázquez and C. Hernández, J. Mod. Optics 521161 (2005). 
"Special Issue in Physiological Optics"

[11] R. M. Goldstein, H. A. Zerbker and C. L. Werner, Radio Science, 23713 (1988).

[12] C. Illueca, D. Mas, J. Pérez, et al., J. Mod. Optics 48811 (2001).

[13] M. Dubbelman, H.A. Weeber, R.G.L. Van Der Heijde, et al., Acta Ophtalmol. Scand. 80379 (2002).

[14] J. Espinosa, J. Rouarch, J. Perez, et al., Optik, in press (2007).

[15] David Mas, Jorge Pérez, Carlos Illueca, et al., Proc. Of SPIE 5959199 (2005).

[16] W.H. Southwell, J. Opt. Soc. Am. A 17 (1981).

[17] D. Mas, J. Pérez, C. Hernández, et al., Optics Commun. 227245 (2003).

[18] J. Pérez, D. Mas, J.J. Miret, et al., Optik 11649 (2005).

[19] David Mas, Julián Espinosa, Jorge Pérez and Carlos Illueca, J. Mod. Optics 53259 (2006).

[20] D. L. Fried, J. Opt. Soc. Am. A 367 (1977).

[21] D. Ghiglia and L. Romero, J. Opt. Soc. Am 11107 (1994).

[22] B. Friedlander and J.M. Francos, IEEE Trans. Signal Processing, 142999 (1996).

[23] Z.P. Liang, IEEE Trans. Med. Imag., 15893 (1996).

[24] S. Dupont, D. Labrousse and M. Berthod, SAR interferometry : An application of simulated annealing to phase unwrapping, Proc. $9^{\text {th }}$ Scandinavian Conf. Image Analysing, 697 (1995).

[25] J.M. Huntley and J. R. Buckland, J. Opt. Soc. Am. A 121990 (1995).

[26] K. Itoh, Appl. Opt. 212470 (1982).

[27] Thomas O. Salmon and Corina van de Pol, J. Cataract Refract Surg 322064 (2006). 
"Special Issue in Physiological Optics"

\section{FIGURE CAPTIONS}

Figure 1 Schematic representation of the human eye.

Figure 2 Central region of the phase field that correspond to the eye 1, a) wrapped phase, b) reconstructed phase.

Figure 3 Topographies of a) eye 1 and b) eye 4 .

Figure 4 Absolute Zernike coefficients for the five eyes. 


\begin{tabular}{|c|c|c|c|c|c|}
\hline $\begin{array}{c}\text { Zernike } \\
\text { Term }\end{array}$ & Eye 1 & Eye 2 & Eye 3 & $\begin{array}{c}\text { Eye } 3 \\
\text { post }\end{array}$ & Eye 4 \\
\hline$Z(3,-3)$ & -0.0332 & -0.0531 & -0.0130 & -0.1063 & 0.7093 \\
\hline$Z(3,-1)$ & -0.0265 & 0.0144 & 0.0524 & 0.1845 & -1.1985 \\
\hline$Z(3,1)$ & 0.0364 & 0.046 & -0.0818 & -0.0454 & -0.1446 \\
\hline$Z(3,3)$ & -0.0743 & 0.0105 & -0.0936 & -0.2467 & 0.0847 \\
\hline$Z(4,-4)$ & 0.1066 & 0.0846 & -0.0015 & -0.024 & 0.0694 \\
\hline$Z(4,-2)$ & -0.0128 & -0.0012 & 0.0108 & 0.0441 & 0.1146 \\
\hline$Z(4,0)$ & -0.0288 & -0.0362 & -0.0380 & 0.1174 & -0.0951 \\
\hline$Z(4,2)$ & -0.0004 & -0.0256 & 0.0354 & 0.0745 & 0.1273 \\
\hline$Z(4,4)$ & 0.0432 & 0.0382 & 0.0539 & 0.1516 & -0.1516 \\
\hline$Z(5,-5)$ & -0.0044 & 0.0096 & 0.0271 & 0.0356 & -0.0288 \\
\hline$Z(5,-3)$ & 0.0008 & 0.0056 & -0.0126 & 0.0048 & -0.0407 \\
\hline$Z(5,-1)$ & 0.0053 & -0.0155 & -0.0020 & -0.0285 & 0.0894 \\
\hline$Z(5,1)$ & 0.0043 & 0.0043 & 0.0007 & -0.0341 & 0.0139 \\
\hline$Z(5,3)$ & 0.0058 & -0.0023 & 0.0068 & 0.0213 & -0.0114 \\
\hline$Z(5,5)$ & 0.0009 & 0.0094 & -0.0033 & -0.0024 & 0.0183 \\
\hline
\end{tabular}

Table 1. Zernike coeffients $(\mu \mathrm{m})$ for $3^{\text {rd }}$ through $5^{\text {th }}$ order aberrations. 
"Special Issue in Physiological Optics"

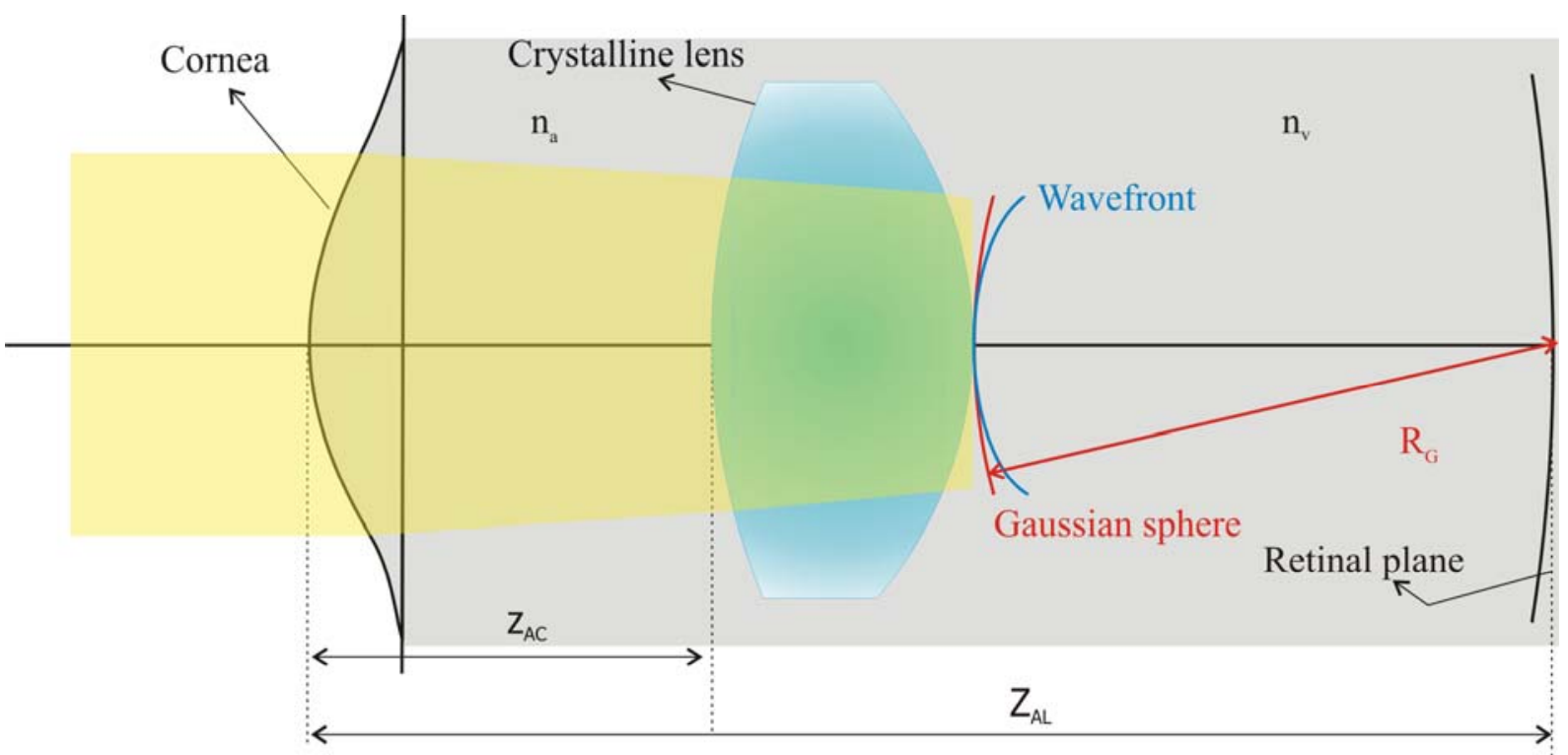

Figure 1. 

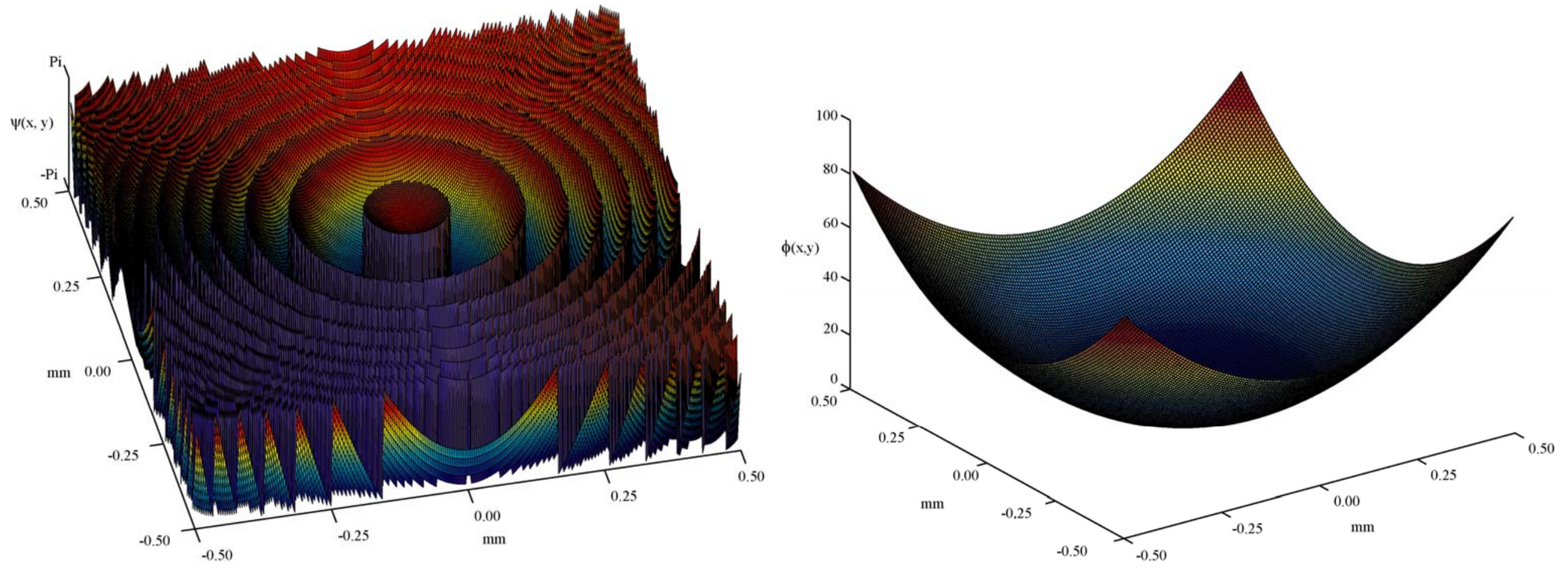

(a)

(b)

Figure 2. 
"Special Issue in Physiological Optics"

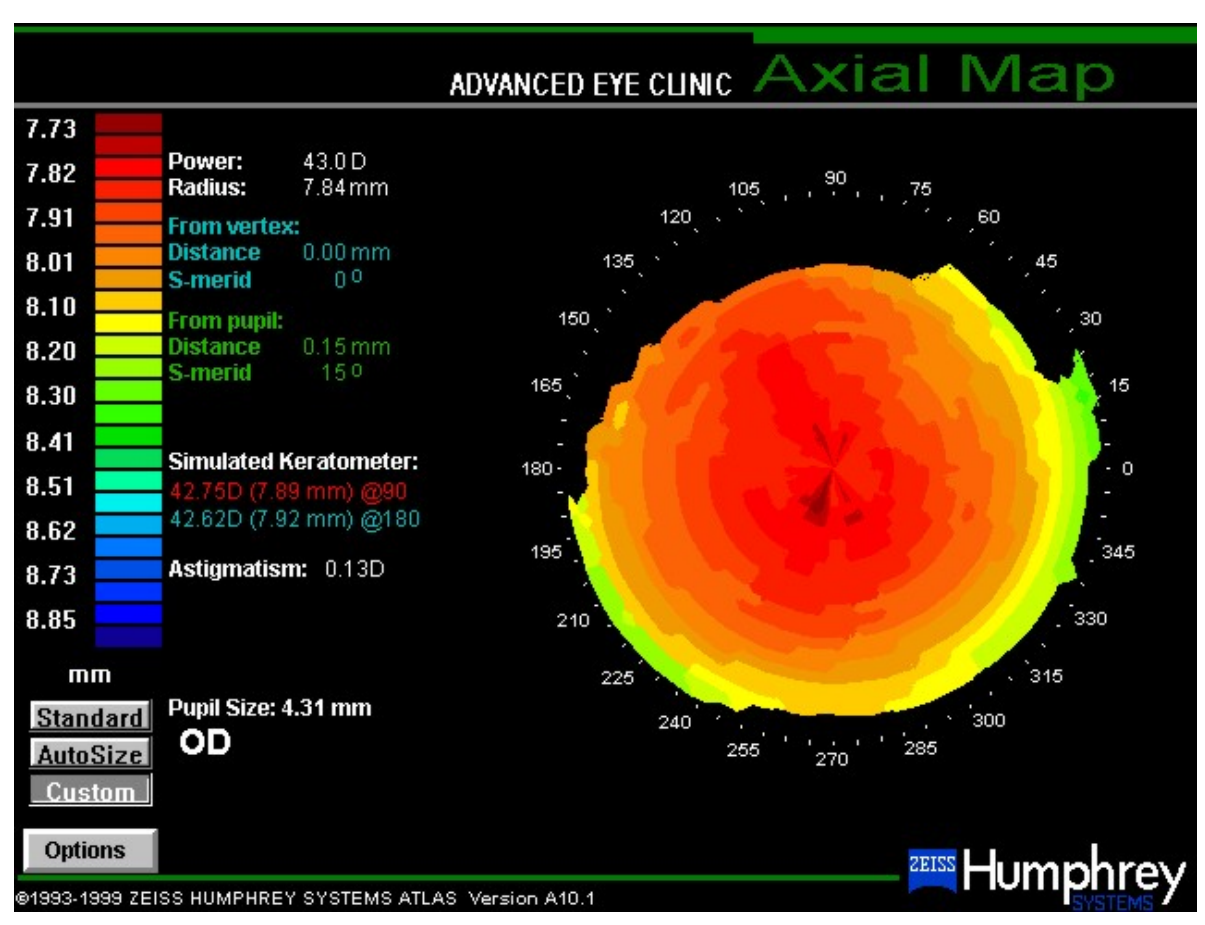

(a)

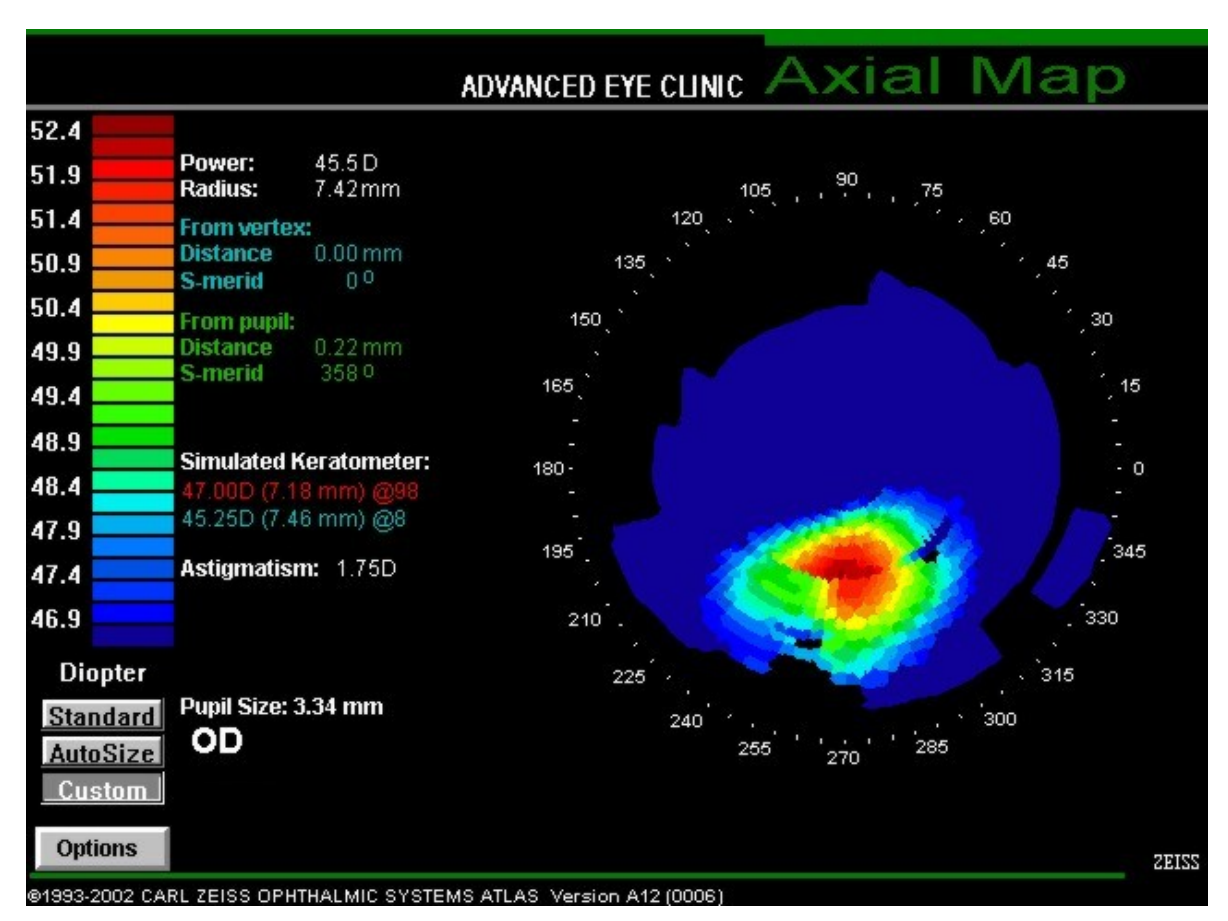

(b)

Figure 3. 
"Special Issue in Physiological Optics"

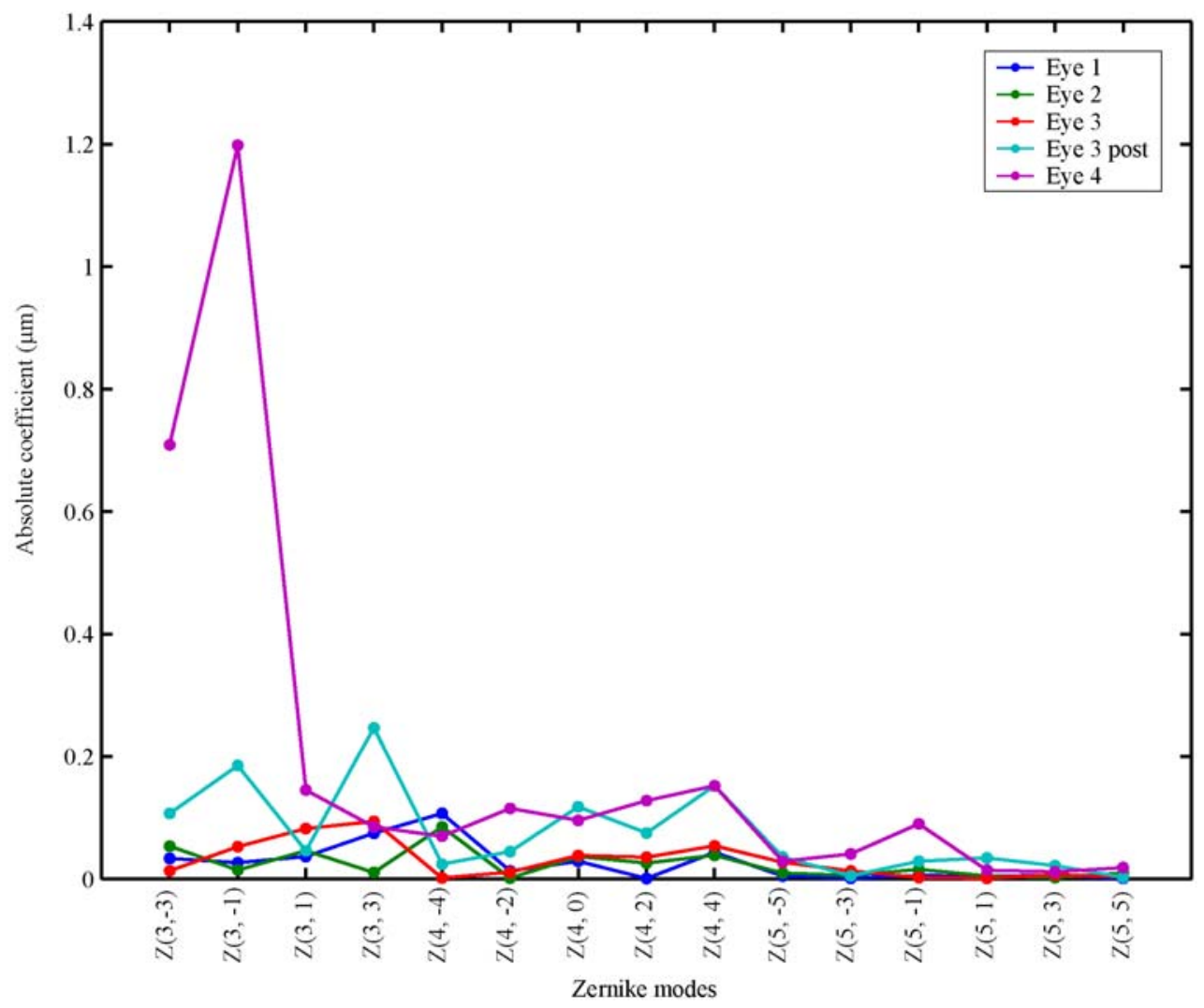

Figure 4 